\title{
Piet W. N. M. van Leeuwen and John C. Chadwick: Homogeneous Catalysis: Activity, Stability and Deactivation
}

\author{
Wiley, Weinheim, 2011, ISBN: 978-3-527-32329-6, Hardback. Copyright 2011, 418 pp
}

\author{
Ricardo Castarlenas
}

Received: 11 March 2013/Accepted: 20 April 2013/Published online: 30 April 2013

(C) Springer Science+Business Media New York 2013

We are not faced herein with the typical academic survey about homogeneous catalysis and issues thereof, which do not entail a decrease of its interest and quality. Its singularity comes from the authors' approach. It is very common that books dealing with this subject put the accent on catalysts preparation, mechanisms, or catalytic activity and selectivity but often tiptoe around catalysts stability and deactivation pathways. However, as clearly highlighted in this volume by long-experienced Profs. Piet van Leeuwen and John Chadwick, it is essential to advance towards the understanding of deactivation processes in order to avoid them and thereby achieve a better efficiency in catalytic transformations.

Covering the current state of different branches of homogeneous catalysis, such as olefin oligomerization or polymerization, asymmetric hydrogenation, carbonylations, cross-coupling reactions or olefin metathesis, the book is of wide interest to researchers, teachers as well as students whose work involves innovative and sustainable chemistry. The first chapter provides the reader with a basic portrait of elementary steps leading to decomposition of the metal complexes and the ligands, with special care taken towards phosphine- or carbene-metal species, together with the introduction of activation and deactivation key concepts. This profuse compilation is really valuable as such type of information is rarely reviewed and thus difficult to find within the literature.

Chapters 2-6 are devoted to industrially relevant polymerization and oligomerization of olefins. The first two chapters of this part of the book are fairly descriptive and organized by the type of metal within each catalyst. More in the line of the books central motif are chapters 4 and 5 dealing with benefits and drawbacks of catalyst inmobilization or the formation of dormant species and their influence one catalytic activity, respectively. Chapter 6 presents recent advances on oligomerization of olefins both to early and late metal promoters, including some methods to avoid catalysts deactivation.

The second part of the volume returns back to the central aim of the book. Certainly, rather exclusive in its approach is chapter 7 about asymmetric hydrogenation. It is amazing to realise the great amount of possibilities that a catalytic process has to breaking bad but at least it is gratifying to find them tabulated at a glance. The remaining three chapters continue on the same philosophy of concisely outlining the subject and profusely describing deactivation pathways on hydroformylation, cross-coupling and olefin metathesis.

The text is well-arranged thorough the monograph. The clear style makes it easy to read, further supported by the very informative formulas and figures. It is highly recommended for experts and novices and should serve as a primary source of valuable information not only for academics but also for industrial researchers. This very particular book should be an essential addition to any meaningful institutional library collection.
R. Castarlenas $(\bowtie)$

Departamento de Química Inorgánica, Facultad de Ciencias,

Universidad de Zaragoza, Zaragoza, Spain

e-mail: rcastar@unizar.es 